\title{
Response to: Combined treatment of intravitreal bevacizumab and intravitreal triamcinolone in patients with retinal vein occlusion by Schroff et al.,
}

\author{
Rita Ehrlich • Thomas A. Ciulla • Adam Moss • \\ Alon Harris
}

Received: 25 February 2010 /Accepted: 27 February 2010/Published online: 30 March 2010

(C) Springer-Verlag 2010

\section{Dear Editor,}

We thank the authors for their interest in our study. We agree that combined treatment with triamcinolone could reduce the number of recurrent treatments with bevacizumab. However, the combination treatment did not seem to change the visual acuity outcome compared to previously published reports with bevacizumab monotherapy. The reduced number of re-injections with the combined treatment need to be weighed against the side-effects after triamcinolone injec- tions, including increased intraocular pressure, cataract, and possible increased risk of endophthalmitis and sterile inflammation.

Looking back at the data, we did not find any difference in the response of previously treated patients compared to treatment-naive eyes, although our study is limited by the small number of patients and relatively short follow-up period. A larger randomized controlled study involving combination therapy is warranted.

R. Ehrlich $(\bowtie) \cdot$ T. A. Ciulla $\cdot$ A. Moss $\cdot$ A. Harris

Department of Ophthalmology,

Indiana University School of Medicine,

702 Rotary Circle,

Indianapolis, IN 46202, USA

e-mail: ritaehrlich@gmail.com 